\title{
O manejo pós operatório do implante do valvar aórtico percutâneo com uso de anticoagulantes e antiagregantes plaquetários: Uma revisão de literatura
}

\author{
The postoperative management of percutaneous aortic valve implantation with the use of \\ anticoagulants and platelet antiaggregants: A review of the literature \\ Manejo postoperatorio de la implantación de la válvula aórtica percutánea con anticoagulantes y \\ antiagregantes plaquetarios: Una revisión de la literatura
}

Recebido: 30/04/2021 | Revisado: 21/05/2021 | Aceito: 25/05/2021 | Publicado: 10/06/2021

\author{
Marlilia Moura Coelho Sousa \\ ORCID: https://orcid.org/0000-0002-5620-3400 \\ Instituto de Educação Superior do Vale do Parnaíba, Brasil \\ E-mail: marliliasousa99@gmail.com \\ Israel Nunes Bezerra \\ ORCID: https://orcid.org/0000-0001-8823-751X \\ Instituto de Educação Superior do Vale do Parnaíba, Brasil \\ E-mail: israelnunes.13@gmail.com \\ Fábio Dias Nogueira \\ ORCID: https://orcid.org/0000-0001-7303-1180 \\ Instituto de Educação Superior do Vale do Parnaíba, Brasil \\ E-mail: fdnvaq@gmail.com \\ Leiz Maria Costa Veras \\ ORCID: https://orcid.org/0000-0003-3422-2878 \\ Instituto de Educação Superior do Vale do Parnaíba, Brasil \\ E-mail: leiz.vera@iesvap.edu.br \\ Daniela Machado Bezerra \\ ORCID: https://orcid.org/0000-0003-3511-4836 \\ Instituto de Educação Superior do Vale do Parnaíba, Brasil \\ E-mail: danielamachadobezerra@ hotmail.com
}

\begin{abstract}
Resumo
Introdução: Um dos principais tratamentos da estenose aórtica pode ser feito através de um procedimento cirúrgico, de "peito aberto", a substituição cirúrgica de valva aórtica (SAVR). Contudo, pacientes classificados como alto risco e/ou com sintomática grave são impossibilitados de realizá-la, diminuindo a sua sobrevida. Devido a isso, surgiram alternativas minimamente invasivas tal como o implante de válvula aórtica transcateter (TAVI). Um dos manejos do pós operatório desse paciente são com terapia de anticoagulantes orais. Objetivo: Conhecer os principais anticoagulantes utilizados no pós operatório da TAVR. Metodologia: Trata-se de estudo descritivo, do tipo revisão de literatura, através das seguintes bases de dados: Scielo, Pubmed e Science Direct. Resultados: Os artigos buscaram esclarecer a fisiopatologia da estenose aórtica, bem como o seu diagnóstico e tratamento. Estes artigos permitiram identificar o mecanismo de ação dos anticoagulantes utilizados no pós-operatório da TAVR. Conclusão: A síntese do conhecimento acerca do manejo da terapia dos anticoagulantes pós operatório da TAVR poderá subsidiar ações dos profissionais médicos a fim de evitar acontecimentos não fisiológicos.
\end{abstract}

Palavras-chave: Anticoagulantes; Estenose aórtica; Implante de prótese de valva cardíaca.

\begin{abstract}
Introduction: One of the main treatments of aortic stenosis can be done through a surgical procedure, "open chest", the aortic valve replacement surgery (SAVR). However, patients classified as high risk and/or with severe symptoms are unable to undergo it, decreasing their survival. Because of this, minimally invasive alternatives have emerged, such as transcatheter aortic valve implantation (TAVI). One of the postoperative management of this patient is with oral anticoagulant therapy. Objective: To know the main anticoagulants used in TAVR postoperative period. Methodology: This is a descriptive study, of the literature review type, through the following databases: Scielo, Pubmed and Science Direct. Results: The articles sought to clarify the pathophysiology of aortic stenosis, as well as its diagnosis and treatment. These articles allowed us to identify the mechanism of action of anticoagulants used in the postoperative period of TAVR. Conclusion: The synthesis of knowledge about the management of anticoagulant therapy after TAVR may support actions of medical professionals in order to avoid non-physiological events.
\end{abstract}

Keywords: Anticoagulants; Aortic stenosis; Heart valve prosthesis implantation. 


\begin{abstract}
Resumen
Introducción: Uno de los principales tratamientos de la estenosis aórtica puede realizarse mediante un procedimiento quirúrgico, a "tórax abierto", la cirugía de reemplazo de la válvula aórtica (SAVR). Sin embargo, los pacientes clasificados como de alto riesgo y/o con síntomas severos no pueden someterse a ella, disminuyendo su supervivencia. Por ello, han surgido alternativas mínimamente invasivas, como la implantación de la válvula aórtica transcatéter (TAVI). Uno de los manejos postoperatorios de este paciente es con terapia anticoagulante oral. Objetivo: Conocer los principales anticoagulantes utilizados en el postoperatorio de TAVR. Metodología: Se trata de un estudio descriptivo, de tipo revisión bibliográfica, a través de las siguientes bases de datos: Scielo, Pubmed y Science Direct. Resultados: Los artículos buscaban aclarar la fisiopatología de la estenosis aórtica, así como su diagnóstico y tratamiento. Estos artículos permitieron identificar el mecanismo de acción de los anticoagulantes utilizados en el postoperatorio del TAVR. Conclusiones: La síntesis del conocimiento sobre el manejo de la terapia anticoagulante después del TAVR puede apoyar las acciones de los profesionales médicos para evitar eventos no fisiológicos.
\end{abstract}

Palabras clave: Anticoagulantes; Estenosis aórtica; Implantación de prótesis de válvulas cardíacas.

\title{
1. Introdução
}

A estenose aórtica é uma valvopatia que acomete mais pacientes idosos, com 75 anos ou mais, nessa faixa etária a valvopatia se apresenta na sua forma mais grave, e está associada com uma alta taxa de morbidade e mortalidade. O tratamento de primeira escolha para essa enfermidade é um procedimento cirúrgico, de "peito aberto", para a substituição da valva aórtica por uma prótese. No entanto, esse procedimento possui um alto risco cirúrgico, especialmente por se tratar de pacientes com uma faixa etária mais avançada e/ou com várias doenças associadas. Atualmente, um novo procedimento cirúrgico, tem sido considerado uma opção terapêutica, que consiste na implantação por meio de um cateter de uma bioprótese valvar aórtica (transcatheter aortic valve implantation [TAVI]), considerado um procedimento menos invasivo (Lopes et al., 2020).

Os pacientes idosos que são submetidos a este procedimento cirúrgico, cerca da metade apresentam associada a estenose aórtica a fibrilação atrial (FA) como comorbidade clínica, isso faz que seja indicado o uso anticoagulante. Ademais, aproximadamente $20 \%$ dos pacientes submetidos à TARV podem apresentar a formação de trombose ao folheto da válvula aórtica. A FA apresenta um elevado risco de o paciente ter um acidente vascular encefálico (AVE) e é um fator de risco de origem neurológica depois de 48 horas após a cirurgia (Mieghem et al., 2018).

Os tratamentos anticoagulantes após a cirurgia do TAVR devem compensar o risco de complicações tromboembólicas e hemorrágicas. Os estudos atuais propõem uma terapia antiplaquetária dupla - com a associação de AAS (ácido acetilsalić́lico) e de um clopidogrel- como o manejo atual de cuidados pós-operatório com base principalmente na apresentação de um consenso empírico (Mieghem et al., 2018).

O papel desses medicamentos é de impedir a ocorrência de trombose patológica e limitar a lesão por reperfusão, porém devem propiciar uma resposta normal à lesão vascular e limitar o sangramento. A formação de um trombo, a trombose, pode ter sua localização tanto na circulação arterial quanto na circulação venosa. Um trombo de origem do sistema venoso é constituído principalmente de fibrina, de eritrócitos e com poucas plaquetas. Já o tombo do sistema arterial tem um grande quantidade de plaquetas (Ferreira et al., 2016).

O objetivo deste estudo foi oferecer uma visão geral sobre o que há de mais recente sobre o manejo da terapia anticoagulante na TAVI em pacientes submetidos à cirurgia cardíaca devido a estenose aórtica. A ênfase dessa revisão, foi a indicação clínica destes fármacos, bem a sua farmacodinâmica e farmacocinética dos anticoagulantes orais e a avaliação de risco do uso dessa medicação após a cirurgia.

\section{Metodologia}

O presente trabalho trata-se de uma revisão integrativa da literatura, que conforme Galvão (2012), é realizada através da Biblioteca Virtual de Saúde (BVS) nas bases de dados Scielo, Pubmed e Science Direct, buscando responder o seguinte questionamento: Qual manejo da terapia anticoagulante e antiplaquetario no pós operatório da cirurgia cardíaca para para 
implante de cateter de uma bioprótese valvar aórtica (transcatheter aortic valve implantation [TAVI])? Foram encontrados 966 artigos, sendo selecionados 44, sendo feito o cruzamento simultâneo entre os descritores em inglês "Anticoagulants", "estenose aórtica", "transcatheter aortic valve implantation", "Pos operative". Quanto aos critérios de inclusão, introduziram-se artigos escritos na língua portuguesa e inglesa publicados entre 2016 e 2021.Foram selecionados estudos que abordam sobre o tratamento com anticoagulantes em pacientes que foram submetidos a uma cirurgia cardíaca para a implante de cateter de uma bioprótese valvar aórtica, com enfoque nos fatores de risco, etiologia, prevenção, diagnóstico e tratamento. Após a pré-leitura e leitura seletiva dos textos, foram selecionados 22 artigos, nos quais realizou-se uma leitura interpretativa buscando responder à pergunta de pesquisa desta revisão.Também foram incluídos estudos sobre a farmacocinética e farmacodinâmica das medicações antitrombóticas. Em relação aos critérios de exclusão foi dispensado artigos que não fossem voltados para a temática central e não atendessem a pergunta norteadora deste estudo.

\section{Discussão e Resultados}

O estudo PARTNER I2 e o CoreValve High-Risk Study6 compararam a SAVR com o TAVI, foi demonstrado uma maior incidência de complicações vasculares e de acidente vascular encefálico (AVE) associadas a TAVI. Porém, outros ensaios mostraram uma taxa de mortalidade menor no grupo TAVI do que no grupo cirúrgico, evidenciando a necessidade de mais pesquisas sobre o assunto. O consenso atual, embasado nas literaturas pesquisadas, afirma que o TAVI seja reservado para pacientes que preencham as indicações para SAVR padrão, porém definidos como de alto risco operatório, contraindicados para cirurgia convencional. Excepcionalmente, pacientes de risco moderado podem ser elegíveis.

O tratamento com anticoagulantes é fundamental na prevenção de acontecimentos tromboembólicos. seu mecanismo de ação funciona de forma a impedir a formação de trombos, garantindo uma resposta normal à lesão vascular, limitando o sangramento (Plácido, 2016).

Os estudos apresentaram que a trombose valvar pode ser mais frequente na substituição de valvar mitral, quando comparado a troca da valvar aórtica, isso também pode ocorrer na substituição válvula aórtica por transcateter. Alguns estudos apontaram, que não foi evidenciado casos de trombose valvar, no entanto o prosseguimento clínico dos pacientes foi em um período de tempo muito curto, cerca de 6 meses, e avaliação por exames de imagem, como a ecocardiográfica foi limitada a 1 mês após o procedimento cirúrgico. Outros estudos apontaram que não há um tipo de tratamento com anticoagulantes ou antiplaquetária utilizada após esse procedimento cirúrgico, que atualmente não há uma padronização de como deve ser realizado o manejo dos antitrombóticos prescritos após este procedimento cirúrgico (Calça et al., 2019).

O número de pacientes que apresentaram no pós-operatório da valva aórtica por transcateter, a trombose como uma complicação após um procedimento cirúrgico foi cerca de 10 casos em 1 ano, conforme foi apresentado um estudo. Ademais houve um aumento significativo em paciente sem nenhum tipo de tratamento com anticoagulantes, do que aqueles que realizaram a terapêutica com os anticoagulantes. Desse modo, recomenda-se que seja feita uma avaliação por imagem, através do ecocardiograma, de forma periódica, e caso seja constatada alguma alteração nos gradientes transproteticos, a suspeita de trombose deve ser confirmada através de uma tomografia computadorizada (TC) de tórax (Lopes et al., 2020).

O tratamento da trombose pode ser realizado através dos anticoagulantes, que são caracterizados com uma classe de fármacos utilizados no tratamento da disfunção da homeostasia sanguínea, assim como os medicamento que são inibidores plaquetários, inibidores trombolíticos e os agentes anti-hemorrágicos (Collet, 2018).

O mecanismo de ação da terapia anticoagulantes pode ser dividida em duas formas: a de ação direta e a de ação indireta. Os anticoagulantes que tem o mecanismo ação direto, na qual são caracterizados por fármacos capazes de inibir a cascata de coagulação, já os anticoagulantes que possuem o mecanismo de ação indireto são os fármacos que agem através da correlação com outras proteínas ou vias metabólicas alterando a fisiologia da cascata de coagulação (Plácido, 2016). Para 
manter o fluxo sanguíneo de forma adequada, devem ocorrer quatro fases inter-relacionadas que são: as fases vascular, plaquetária, da coagulação e fibrinolítica (Ramos, 2020).

Entre os processos químicos e biológicos que permeiam o uso de um fármaco estão a farmacocinética e a farmacodinâmica. A farmacocinética aborda sobre os efeitos do agente farmacológico pelo corpo, incluindo seus mecanismos de absorção, distribuição, metabolização e excreção. Sobre os anticoagulantes, sua via da administração é de preferência oral, contudo hoje temos as vias parenteral, subcutânea ou endovenosa, tendo efeito mais rápido se comparado com a oral (Izar, 2017).

A principal complicação da anticoagulação é o sangramento, tendo em vista que procedimentos que apresentam um risco significativo de sangramento significativo podem exigir a suspensão temporária do medicamento (Collet, 2018).

O regime antiplaquetário/anticoagulante ideal após o implante transcateter de válvula aórtica (TAVI) ainda permanece controverso pois precisa equilibrar o risco entre complicações tromboembólicas e hemorrágicas. As diretrizes atuais revisadas nesse estudo recomendaram a utilização de aspirina + clopidogrel por 3 a 6 meses depois do TAVI seguida por aspirina em monoterapia, em pacientes que não tem uma indicação formal para anticoagulação oral (ex: fibrilação atrial, tromboembolismo venoso, etc). (Brouwer, 2020)

A terapia antiplaquetária dupla (DAPT) continua sendo a melhor estratégia após o implante transcateter da válvula aórtica (TAVI). Contudo, alguns estudos sugeriram que a terapia antiplaquetária única (SAPT) constituindo apenas da aspirina pode ser uma alternativa. (Rodés-Cabau, 2017)

A rivaroxabana, um inibidor direto do fator Xa demonstrou reduzir o risco de tromboembolismo em diferentes contextos clínicos na dose diária de $10 \mathrm{mg} /$ dia na prevenção do tromboembolismo venoso.

O GALILEO, ensaio clínico randomizado e controlado, foi realizado para avaliar a eficácia e segurança da terapia antitrombótica à base de rivaroxabana $10 \mathrm{mg} / \mathrm{dia}$ em comparação com a terapia antiplaquetária após TAVR bem-sucedido em pacientes sem indicação estabelecida para anticoagulação para prevenir tromboembolismo de superfície valvar enquanto mitiga complicações hemorrágicas. A rivaroxabana foi associada a menor incidência de espessamento do folheto da valva aórtica e redução do movimento do folheto do que a terapia antiplaca. (Dangas, 2019).

\section{Conclusão}

A presente revisão de literatura sugere que a seja associado no tratamento do pós-operatório da TAVI o uso de anticoagulantes e antiplaquetários, com uma forma de prevenção da formação de trombos dessas estruturas anatômicas - valva aórtica. Assim, há necessidade de mais pesquisas para elucidar o manejo correto desses medicamentos - quanto a sua farmacodinâmica e sua farmacocinética - a fim de se padronizar a terapêutica correta nesse procedimento cirúrgico.

Ademais, sugere-se a realização de mais pesquisas para avaliar a eficácia do uso de outras terapias anticoagulantes e antiplaquetárias para melhorar a sobrevida do paciente submetido ao implante valvar aórtico percutâneo.

\section{Referências}

Blanke, P., Leipsic, J. A., Popma, J. J., Yakubov, S. J., Deeb, G. M., Gada, H., \& Reardon, M. J. (2020). Bioprosthetic aortic valve leaflet thickening in the Evolut low risk sub-study. Journal of the American College of Cardiology, 75(19), 2430-2442.

Brouwer, J., Nijenhuis, V. J., Delewi, R., Hermanides, R. S., Holvoet, W., Dubois, C. L., \& Ten Berg, J. M. (2020). Aspirin with or without clopidogrel after transcatheter aortic-valve implantation. New England Journal of Medicine, 383(15), 1447-1457.

Calça, R., Teles, R. C., Branco, P., Gaspar, A., Brito, J., Nolasco, T., \& Weigert, A. (2019). Impacto do Implante Percutâneo de Válvula Aórtica na Função Renal. Arquivos Brasileiros de Cardiologia, 113(6), 1104-1111.

Celis, D., Gomes, B. A. D. A., Ibanez, I., Azevedo, P. N., Teixeira, P. S., \& Nieckele, A. O. (2020). Predição do Mapa de Estresse em Aorta Ascendente: Otimização da Posição Coaxial no Implante Valvar Aórtico Percutâneo. Arquivos Brasileiros de Cardiologia, (AHEAD). 
Coelho, N. H., Pontes, R. L., Silva, R., Martins, V., Oliveira, C., Campos, J., \& Bentes, C. (2018). INTRAOPERATIVE ANTICOAGULATION MONITORIZATION IN VASCULAR SURGERY-DOES A BLIND DOSIS FITS ALL?. Angiologia e Cirurgia Vascular, 14(4), 301-306.

Collet, J. P., Berti, S., Cequier, A., Van Belle, E., Lefevre, T., Leprince, P., \& Montalescot, G. (2018). Oral anti-Xa anticoagulation after trans-aortic valve implantation for aortic stenosis: the randomized ATLANTIS trial. American heart journal, 200, 44-50.

Dangas, GD, Tijssen, JG, Wöhrle, J., Søndergaard, L., Gilard, M., Möllmann, H., \& Windecker, S. (2020). Um ensaio controlado de rivaroxabana após a substituição transcateter da válvula aórtica. New England Journal of Medicine, 382 (2), 120-129.

Yoshida, R. D. A., Yoshida, W. B., \& Rollo, H. D. A. (2011). Novos anticoagulantes para a profilaxia do tromboembolismo venoso em cirurgias ortopédicas de grande porte. Jornal Vascular Brasileiro, 10(2), 145-153.

De Araújo, W. E. C., \& de Melo Barbosa, A. (2020). Eficácia, segurança e custo-efetividade dos anticoagulantes orais diretos para prevenção de eventos tromboembólicos nos casos de Fibrilação Atrial não Valvar, anticoagulados com varfarina e eventos adversos graves: revisão rápida de evidências. Revista Científica Da Escola Estadual De Saúde Pública De Goiás" Cândido Santiago", 6(1), 113-138.

De Backer, O., Dangas, G. D., Jilaihawi, H., Leipsic, J. A., Terkelsen, C. J., Makkar, R., \& Søndergaard, L. (2020). Reduced leaflet motion after transcatheter aortic-valve replacement. New England Journal of Medicine, 382(2), 130-139.

Fernandes, C. J. C. D. S., Alves Júnior, J. L., Gavilanes, F., Prada, L. F., Morinaga, L. K., \& Souza, R. (2016). Os novos anticoagulantes no tratamento do tromboembolismo venoso. Jornal Brasileiro de Pneumologia, 42(2), 146-154.

Goel, R., Power, D., Tchetche, D., Chandiramani, R., Guedeney, P., Claessen, B. E., \& Dangas, G. D. (2019). Impact of diabetes mellitus on short term vascular complications after TAVR: Results from the BRAVO-3 randomized trial. International journal of cardiology, 297, 22-29.

Jørgensen, T. H., Thyregod, H. G. H., Tarp, J. B., Svendsen, J. H., \& Søndergaard, L. (2017). Temporal changes of new-onset atrial fibrillation in patients randomized to surgical or transcatheter aortic valve replacement. International journal of cardiology, 234, 16-21.

Kosmidou, I., Liu, Y., Alu, M. C., Liu, M., Madhavan, M., Chakravarty, T., \& Leon, M. B. (2019). Antithrombotic therapy and cardiovascular outcomes after transcatheter aortic valve replacement in patients with atrial fibrillation. JACC: Cardiovascular Interventions, 12(16), 1580-1589.

Conrotto, F., D'Ascenzo, F., D'Amico, M., Moretti, C., Pavani, M., Scacciatella, P., \& Nietlispach, F. (2017). Outcomes of patients with low-pressure aortic gradient undergoing transcatheter aortic valve implantation: a meta-analysis. Catheterization and Cardiovascular Interventions, 89(6), $1100-1106$.

Lorga Filho, A. M., Azmus, A. D., Soeiro, A. M., Quadros, A. S., Avezum Junior, A., Marques, A. C., \& Portal, V. L. (2013). Diretrizes brasileiras de antiagregantes plaquetários e anticoagulantes em cardiologia. Arquivos Brasileiros de Cardiologia, 101(3), 01-95.

Meneguz-Moreno, R. A., Castro-Filho, A. D., Ramos, A. I. D. O., Zumarraga, M., Bihan, D. L., Barretto, R., \& Sousa, J. E. (2017). Evolução e Prognóstico da Regurgitação Paravalvar Após Implante de Valva Aórtica Transcateter. Arquivos Brasileiros de Cardiologia, 109(6), 590-598.

Monteiro, C., Ferrari, A. D. L., Caramori, P. R. A., Carvalho, L. A. F., Siqueira, D. A. D. A., Thiago, L. E. K. S., \& Brito Junior, F. S. D. (2017). Marca-passo Definitivo após Implante Valvar Aórtico Transcateter: Incidência, Preditores e Evolução da Função Ventricular Esquerda. Arquivos Brasileiros de Cardiologia, 109(6), 550-559.

Morais, Q. C. D. (2017). Análise de decisão multicritério-MCDA-em hospital terciário: prevenção de trombose venosa profunda em cirurgias ortopédicas de grande porte.

Munari, E., \& Geron, V. L. M. G. (2019). Uso De Heparina Em Pós-Operatório De Cirurgia Bariátrica. Revista Científica da Faculdade de Educação e Meio Ambiente, 10(1), 139-147.

Pitta, G. B. B., Pereira, D. A., Oliveira, M. D. F. Q., Guedes, E. A., \& Sampaio, J. A. (2017). Cateterismo de artéria mesentérica para tratamento de trombose de veia porta. Jornal Vascular Brasileiro, 16(1), 43-47.

Plácido, A. I. P. (2016). Anticoagulantes orais: terapêutica clássica versus novos anticoagulantes (Doctoral dissertation).

Ramos, B. R. S. (2020). Terapêutica com anticoagulantes orais em crianças com substituição de válvula aórtica (Doctoral dissertation).

Rocha, A. T. C., Pinheiro, T. B., Souza, P. R. S. P. D., \& Marques, M. A. (2020). Protocolos de profilaxia de tromboembolismo venoso (TEV) em hospitais

Roque, D., Magno, P., Ministro, A., Santos, M., de Sousa, M., Augusto, J., \& Morais, C. (2018). Tromboembolismo pulmonar e disseção da aorta concomitantes: abordagem à anticoagulação. Revista Portuguesa de Cardiologia.

Roque, D., Magno, P., Ministro, A., Santos, M., de Sousa, M., Augusto, J., \& Morais, C. (2020). Concomitant pulmonary embolism and aortic dissection: An approach to anticoagulation. Revista Portuguesa de Cardiologia (English Edition), 39(6), 351-e1.

Tarantini, G., Mojoli, M., Urena, M., \& Vahanian, A. (2017). Fibrilação atrial em pacientes submetidos ao implante transcateter de válvula aórtica: epidemiologia, tempo, preditores e resultado. European heart journal, 38 (17), 1285-1293.

Rodés-Cabau, J., Masson, J. B., Welsh, R. C., Garcia del Blanco, B., Pelletier, M., Webb, J. G, \& Côté, M. (2017). Aspirina versus aspirina mais clopidogrel como tratamento antitrombótico após a substituição da válvula aórtica transcateter por uma válvula expansível por balão: o ensaio clínico randomizado ARTE (Aspirina versus Aspirina + Clopidogrel após implante de válvula aórtica transcateter). JACC: Cardiovascular Interventions, 10 (13), 1357-1365.

Van Belle, E., Hengstenberg, C., Lefevre, T., Kupatt, C., Debry, N., Husser, O., \& Bravo-3 MRI Study Investigators. (2016). Cerebral embolism during transcatheter aortic valve replacement: the BRAVO-3 MRI study. Journal of the American College of Cardiology, 68(6), 589-599. 
Research, Society and Development, v. 10, n. 6, e58410615631, 2021

(CC BY 4.0) | ISSN 2525-3409 | DOI: http://dx.doi.org/10.33448/rsd-v10i6.15631

Van Mieghem, N. M., Unverdorben, M., Valgimigli, M., Mehran, R., Boersma, E., Baber, U., \& Dangas, G. D. (2018). Edoxaban versus standard of care and their effects on clinical outcomes in patients having undergone transcatheter aortic valve implantation in atrial fibrillation - rationale and design of the ENVISAGE-TAVI AF trial. American heart journal, 205, 63-69.

Whalen, K., Finkel, R., \& Panavelil, T. A. (2016). Farmacologia Ilustrada- (6a ed.). Artmed Editora.

Windecker, S., Tijssen, J., Giustino, G., Guimarães, A. H., Mehran, R., Valgimigli, M., \& Dangas, G. D. (2017). Trial design: rivaroxaban for the prevention of major cardiovascular events after transcatheter aortic valve replacement: rationale and design of the GALILEO study. American heart journal, 184, 81-87. 